\title{
Universiteit
}

Leiden

The Netherlands

\section{Myocardial perfusion imaging: Lessons learned and work to be done- update}

Iskandrian, A.E.; Dilsizian, V.; Garcia, E.V.; Beanlands, R.S.; Cerqueira, M.; Soman, P.; ... ; Decker, W. van

\section{Citation}

Iskandrian, A. E., Dilsizian, V., Garcia, E. V., Beanlands, R. S., Cerqueira, M., Soman, P., ... Decker, W. van. (2018). Myocardial perfusion imaging: Lessons learned and work to be doneupdate. Journal Of Nuclear Cardiology, 25(1), 39-52. doi:10.1007/s12350-017-1093-7

Version: $\quad$ Not Applicable (or Unknown)

License: $\quad$ Leiden University Non-exclusive license

Downloaded from: https://hdl.handle.net/1887/86888

Note: To cite this publication please use the final published version (if applicable). 


\section{Myocardial perfusion imaging: Lessons learned and work to be done-Update \\ By Editors of J Nuc Cardiol}

Ami E Iskandrian, MD, MASNC (1), Vasken Dilsizian , MD, MASNC (2), Ernest V Garcia , PhD, MASNC (3), Rob S. Beanlands, MD, FASNC (4), Manuel Cerqueira, MD, MASNC (5), Prem Soman, MD, PhD, FASNC (6), Daniel S. Berman, MD (7); Alberto Cuocolo, MD (8); Andrew J Einstein, MD, PhD (9); Charity J Morgan, PhD (10); Fadi G. Hage, MD (11); Heinrich R. Schelbert, MD, PhD (12); Jeroen J Bax, MD, PhD (13); Joseph C. Wu; MD, PhD (14); Leslee J Shaw, PhD, MASNC (15); Mehran M. Sadeghi, MD (16); Nagara Tamaki, MD (17); Philipp A. Kaufmann, MD (18); Robert Gropler, MD, MASNC (19); Sharmila Dorbala, MD, MPH, FASNC (20); William Van Decker, MD, MASNC (21)

1-University of Alabama at Birmingham, 2-University of Maryland School of Medicine, 3-Emory University, 4- University of Ottawa Heart Institute , 5-Cleveland Clinic, Lerner College of Medicine, Case Western Reserve University, 6-University of Pittsburgh Medical Center,7-Cedars-Sinai Medical Center, University of California at Los Angeles; 8- University of Naples Federico II; 9- Columbia University; 10- University of Alabama at Birmingham, 11- University of Alabama at Birmingham and Birmingham Veterans Affairs Medical Center, 12- University of California, Los Angeles; 13- Leiden University Medical Center; 14Stanford University; 15--Emory University, 16- Yale University; 17- Hokkaido University; 18- University Hospital, Zurich, Switzerland; 19- Washington University in St. Louis, MO; 20- Harvard University; 21Temple University

Running title: MPI and lessons learned-part 2

Key words: Myocardial perfusion imaging, Positron emission tomography, single photon emission tomography, nuclear imaging, prognosis, coronary artery disease

\section{Correspndance}

Ami E. Iskandrian, MD, MASNC

Editor-in-Chief/ Journal Nuclear Cardiology

Distinguished Professor of Medicine and Radiology,

Division, Cardiovascular Diseases, Department of Medicine,

University of Alabama at Birmingham,

318 LHRB/ 1900 University BLVD, Birmingham, AL 35294,

Phone: 205 934-0545/ Fax: 205 934-7579,

E-mail: aiskand@uab.edu 


\section{Disclosures}

1-Nothing to disclose

2-Nothing to disclose

3- Royalties from sale of medical software, shares of Syntermed

4-Consultant: GE, JubilantDraxImage, Lanthues,; Research Grants from JubilantDraxlmage, Lantheus

5-Consultant: Astellas Pharma USA, GE; Speakers Bureau: Astellas Pharma USA

6-Grant funding to institution: Astellas Pharma; Advisory Board: Alnylam Pharma

7-Software Royalties from Cedars-Sinai Medical Center

8-Nothing to disclose

9-Grant funding to institution: GE Healthcare, Philips Healthcare, Toshiba America Medical Systems. Advisory board: GE Healthcare.

10-Nothing to disclose

11-Grant support: Astellas Pharma, Novartis Pharma.

12-Nothing to disclose

13-The Dept of Leiden University Medical Center, The Netherlands, has received unrestricted research grants from Biotronik, Medtronic, Boston Scientific and Edwards Lifescience

14-Nothing to disclose

15-Nothing to disclose

16-Consultant Bracco Research USA

17-Grant funding to institution: Nihon Medi-Physics Co Ltd. and Fuji-Film RI Pharma Co Ltd.

18-The Department of Nuclear Medicine, University Hospital Zurich holds a Research contract with GE Healthcare

19-Consultant Biomedical Systems

20-Research grant Astellas Pharma; Stockownership GE.

21-Nothing to disclose

\section{Abbreviations}


ACC: American college of cardiology

AHA: American heart association

CABG: Coronary artery bypass grafting

CAC: Coronary artery calcium

CAD: Coronary artery disease

CTA: Computed tomography angiography

DM: Diabetes mellitus

ECG: electrocardiogram

ESC: European society of cardiology

EF: Ejection Fraction

FFR: Fractional flow reserve

HF: Heart failure

LV: left ventricular/left ventricle

LOE: Level of evidence

MBF: Myocardial blood flow

MI: Myocardial infarction

MPI: myocardial perfusion imaging

MRI: Magnetic resonance imaging

MFR: Myocardial flow reserve

$\mathrm{PCl}$ : percutaneous coronary artery intervention

PET: positron emission tomography

PROMISE: Prospective multicenter imaging study for evaluation of chest pain

$\mathrm{RV}$ : Right ventricular/right ventricle

SPECT: single photon emission tomography

SSS: Summed stress score

SRS: Summed rest score

SDS: summed difference score

TID: Transient ischemic dilatation 


\section{Summary}

As the second term of our commitment to Journal begins we, the editor, would like to reflect on a few topics that have relevance today. These include: prognostication and paradigm shifts, Serial testing: How to handle data? Is the change in perfusion predictive of outcome and which one? Ischemia guided therapy: fractional flow reserve vs. perfusion vs. myocardial blood flow; positron emission tomography (PET) imaging using Rubidium-82 vs. N-13 ammonia vs. F-18 Flurpiridaz; How to differentiate microvascular disease from 3-vessel disease by PET? The imaging scene outside the United States, what are the differences and similarities? Radiation exposure; Special issues with the new cameras? Is attenuation correction needed? Are there normal databases and are these specific to each camera system? And finally, hybrid imaging with single photon emission tomography or PET combined with computed tomography angiography or coronary calcium score. We hope these topics are of interest to our readers.

In 2014, we published an inaugural paper listing some of the themes and challenges in nuclear cardiology (1). Table 1 is a summary of the topics addressed in that paper. In the interim, the Journal of Nuclear Cardiology has published numerous papers dealing with these and many more issues and for that we are very pleased. While many of these topics have been addressed, few, [such as how do we integrate nuclear and nonnuclear data in guiding patient management on a personalized basis? (Items \#6) and 'what is the model nuclear cardiology laboratory in the next decade?" (Item \#15)] need more work and input. We believe our reports could be more a bit more comprehensive, reader-friendly, and personalized and the configuration of nuclear labs in the future may vary depending on resources and needs and there may differences between academic institutions and out- patient imaging facilities.

The journal has published several papers on the expanding use of nuclear cardiology in nonischemic heart diseases such as sarcoid and amyloid heart diseases, infections of native valves, prosthetic valves and pacemaker or defibrillator leads and pockets and neuro-imaging to mention few.

As the Journal begins a second term under same editorship, we thought to present the second inaugural paper in which we identify a new set of challenges. Much has been written about these topics but our goal is to provide a broad perspective based on the experience and knowledge of our seasoned editors.

We think to move forwards we need to learn from our past, both success stories and failures. More on this could be found in the Editor's page in this issue, Amongst the many Nobel laureates in our field, are Marie Curie (MC) and her husband Pierre Curie. Every practitioner should know of them, since $\mathrm{Ci}$ and $\mathrm{mCi}$ are commonly used measurements in the field of nuclear medicine. Ironically, MC turned down the first marriage proposal by Pierre but accepted his second, in which he wrote" it would be a beautiful thing, a thing I dare not hope, if we could spend our life near each other hypnotized by our dream: your patriotic dream, our humanitarian dream and our scientific dream"; so sweet. MC won 2 Nobel prizes

Seventy years after $\mathrm{MC}$ won the first of two Nobel Prizes, a seminal paper on myocardial perfusion imaging (MPI) was published in the New England Journal of Medicine (2). Of note, the summary (abstract) of this paper was not structured; there were only 43 patients ( 15 of them had rest studies only); the MPI was done using planar imaging with a radionuclide that is no longer in clinical use, and there were only 
simple statistical analyses! The authors of this paper were from the Travis Airforce Base. Yet this paper changed the careers of many of us.

In 2017 , the Journal established a committee to identify and acknowledge individuals who had or those who continue to provide meaningful contributions in our field (3)

\section{Prognostication and paradigm shifts:}

The use of MPI has changed over time from establishing diagnosis of coronary artery disease (CAD) to risk assessment and decision making for appropriate treatment strategies. Prediction of patient outcome has also changed over time from short-term outcome (warranty period) to long-term outcome. Outcome has been defined as death (sudden death, cardiac death, and all -cause mortality); non-fatal myocardial infarction (MI); heart failure (HF) or HF exacerbation, the need for hospitalization; unstable angina, the need for coronary angiography or revascularization, quality of life or change in medications. As the bar was raised up further, new items became even more important such as ability to alter outcome using MPI, the need to demonstrate value (value =outcome achieved relative to cost),-Could the risk be modified? How? Is risk modification predictable by changes using serial imaging? (4)

Figure 1 shows images from 2 patients, one with a reversible defect (ischemia) and one with a persistent (fixed) defect of comparable size and distribution. The summed stress score (SSS) is similar in the 2 patients but not the sunned difference score (SDS) and summed rest score (SRS). These images raise several issues in terms of assessing diagnosis and prognosis. First, should patients with prior MI (and fixed defects) be included in assessing diagnostic accuracy of MPI? One can argue this point both ways, because surely fixed defects are indicative of CAD (in most patients) but it also inflates sensitivity. But the bigger question is "which of the prognostic endpoints is most applicable to the two patients"? Often, the 2 patients are lumped together but the question should we couple image findings to specific outcome (s)? Intuitively, one would think that the likely events in the first patient are myocardial infarction, unstable angina or death while in the second patient are HF or death This issue is not unique to MPI but apply to all imaging modalities.

Figure 2 shows serial images on a patient with CAD and angina pectoris. At baseline, there is a moderate sized stress-induced perfusion defect. After medical therapy, symptoms improved and there was a decrease in perfusion defect size (reversible defect). When therapy was discontinued, the symptoms worsened and there was an associated increase in defect size to pretreatment level. So far so good but interestingly, the level exercise, as defined by METS and peak heart rate, were identical on all 3 occasions. So, the question is which endpoint is most useful in this patient? Did changes in perfusion defect explain the symptomatic improvement? Is it possible that the perfusion abnormality occurred early during exercise before treatment (silent ischemia) but late during exercise after treatment? These questions also highlight the difficulty in translating data derived from a large cohort of patients to individual patient. Finally, in the current era of cost containment was serial imaging in this patient cost-effective?

There is also a paradigm shift in imaging because CAD mortality rates have decreased by $70 \%$ in the past 20 years, $\mathrm{MI}$ is less frequent and less severe, there is a decline in frequency of inducible ischemia by stress SPECT MPI, the notion that MPI underestimates extent of CAD and has limited ability to detect subclinical atherosclerosis, the shift in nuclear imaging from being a revenue center to being a cost center, and increasing competition from other imaging modalities. These changes have resulted in examining the 
need of alternative methods such as the coronary calcium scoring in asymptomatic patients and hybrid imaging in symptomatic patients (see below) (5).

\section{Serial testing: How to handle data? Is the change predictive of outcome and which one?}

There are several issues with serial testing. For simplicity, we will focus on MPI. When is it indicated? How is it performed and analyzed? How reproducible are the results so that serial changes can be accurately assessed? Each one of these issues deserve a full review, and some of it has been discussed previously (1). There are few observations worth noting; First, none of the guidelines and appropriateness criteria refer to the need or recommend serial imaging; one would think that there are subgroups of patients where this might be useful, such as patients with silent ischemia, patients with a large area of ischemia who are treated medically, or those with persistent symptoms despite undergoing successful coronary artery revascularization to mention a few. Second, despite 4 decades of using MPI and thousands of publications, the number of papers on serial imaging is small. This may have different reasons but one that comes to mind is the difficulty of how to analyze the data?

Two recent papers, one on coronary calcium scoring and one on troponin, addressed serial imaging $(6,7)$. The calcium paper found that the final calcium score but not the change in calcium score from the first to the second study, to be the most important marker of outcome (6). On the other hand, the troponin paper found the change to be the important marker (7). The question is why? Is it because troponin measurements are more reproducible than calcium score measurements or because an increase in coronary calcium may represent plaque healing?

With MPI, the problem is even greater as the options in interpretation are more diverse: initial vs. follow-up vs. changes in SSS, SDS and SRS. Keep in mind that worsening in one vascular territory and improvement in another could result in no net change! That may be true even when the analysis is performed on a regional rather than global level

This observation raises the question of what is the ideal method of analysis? Surely "supervised" automated analysis is preferred over visual analysis because of a better reproducibility, but the conventional method of automated analysis involves comparing each set of images (initial and follow-up) to a normal database. Recently a method has been described where multiple images from the same patient are compared to each other rather than to a database, which is appealing and deserves to be tested in future studies because smaller or milder differences might be more readily detected (8).

The use of serial MPI is inherently more difficult than serial radionuclide angiography where the LV ejection fraction (EF) is the measurement of interest. There are data on serial testing using phase analysis and neuro-imaging but the settings in which serial testing provides value using these methods need to be addressed $(9,10)$.

\section{Ischemia guided therapy: fractional flow reserve vs. perfusion vs. MBF}

Stress-induced (exercise or pharmacologic) reversible myocardial perfusion defects have been firmly established to reflect myocardial ischemia in a vascular territory that is often subtended by a flowlimiting epicardial coronary artery stenosis (11). These MPI studies are interpreted primarily by assessing 
regional perfusion defects in relative terms, qualitatively or semi-quantitatively. Physiological studies of the coronary circulation and regulation of myocardial blood flow (MBF) are optimally performed with measurements of absolute MBF $(\mathrm{mL} \cdot \mathrm{min}-1 \cdot \mathrm{g}-1)(12)$. Several retrospective prognostic studies have reported on the incremental prognostic value of absolute MBF and flow reserve with positron emission tomography (PET) compared to the conventional relative radiotracer uptake. An important driver for cardiac PET in this direction comes from recent trials which have suggested that physiological indices are superior to anatomical ones for guiding therapy and improving outcomes in CAD patients (13). Unlike so many facets of modern cardiovascular practice and CAD management, where absolute values of key parameters and indices are measured, absolute rest and maximal MBF have yet to be incorporated into routine clinical practice (12-15). There are numerous reports of absolute PET MBF measurements identifying multi-vessel $C A D$ that were missed when images were interpreted in terms of relative radiotracer uptake (16). However, systematic comparison of absolute PET MBF measurements with SPECT MPI for the diagnosis of CAD has not been reported.

Recent efforts in the interventional laboratory have focused on coronary pressure-based measurements such as fractional flow reserve (FFR) to assess maximal coronary vasodilator capacity and flow reserve as indicators of coronary stenosis severity and microvascular function. FFR is an invasive measurement of the coronary circulation and has gained wide acceptance as a decision-making tool for coronary revascularization because it is an excellent predictor of clinical outcomes (17). Non-invasive measurements of absolute MBF with PET are equally suited for determining the physiologic significance of CAD with the advantage of preventing unnecessary cardiac catheterization in patients who may not benefit from revascularization. In one report, applying rubidium-82 PET, the threshold of stress MBF $<0.91$ $\mathrm{mL} \cdot \mathrm{min}^{-1} \cdot \mathrm{g}^{-1}$ was shown to be highly predictive of the occurrence of severe myocardial ischemia (16). In contrast, evidence of severe ischemia was rarely encountered when the stress MBF was $>1.12 \mathrm{~mL} \cdot \mathrm{min}^{-1}$.

Studies comparing MBF by PET to FFR have shown only modest correlation between FFR and coronary flow reserve ratio (CFR, stress/rest MBF) because of the divergence of peak MBF from FFR in the range from 0.7 to 1.0 , due to presence or absence of diffuse and/or microvascular disease. A much stronger correlation with relative flow reserve (stress flow in culprit zone/rest flow in culprit zone divided by stress flow in control zone/rest flow in control zone) has been reported (18). The correlation of relative flow reserve with FFR likely reflects the fact that FFR had initially been validated against stress induced defects on MPI.

Accordingly, it has been hypothesized that patients manifesting ischemia with pharmacologic vasodilator stress in whom MBF cannot be increased $>0.91 \mathrm{~mL} \cdot \mathrm{min}^{-1} \cdot \mathrm{g}^{-1}$ may be at high risk for cardiovascular death or $\mathrm{Ml}$ and would benefit most from coronary revascularization (16). While these thresholds need to be tested and verified in multicenter clinical trials, it is important to keep in mind that decisions about the need for cardiac catheterization and coronary revascularization must be individualized and cannot be made solely on a single stress MBF or flow reserve value. Moreover, there will be individual variations in MBF required to achieve a given level of external work that is free of myocardial ischemia (19). A desirable change in nuclear cardiology is to bring noninvasive absolute PET MBF measurements in line with coronary interventional practice, which is increasingly focused on objective, functional parameters of CAD severity, to guide management of myocardial ischemia and to predict and improve patient outcomes $(12,20)$. 


\section{PET imaging. Rubidium-82 vs. $\mathrm{N}-13$ ammonia vs. F-18 Flurpiridaz}

Currently N-13 ammonia and rubidium-82 (Rb-82) are approved by the Food and Drug Administration in the Unites States for assessing myocardial perfusion and MBF. Oxygen-15 water is also used, but less frequently. F-18 Flurpiridaz is undergoing clinical trials with the hope of future approval.

F-18 Flurpiridaz has a longer half-life than N-13 ammonia and Rubidum-82 (109 min vs. 9.96 min vs $1.25 \mathrm{~min}$, respectively) and requires a regional cyclotron for its production rather than an on-site cyclotron ( $\mathrm{N}-13$ ammonia) or generator (Rubidium-82). Further, the positron range of F-18 Flurpiridaz is shorter than that of $\mathrm{N}-13$ ammonia and Rubdium-82 (1.03 vs $2.53 \mathrm{vs.} 8.6 \mathrm{~mm}$, respectively), which means a higher spatial resolution of the perfusion images. Also, the initial extraction fraction is higher than $\mathrm{N}-13$ ammonia and Rubidium-82 and closer to Oxygen-15 water even at high rates of MBF. The longer half-life of F-18 enables the use of F-18 Flurpiridaz in virtually all centers that provide PET scanning, without the need for sufficient volume of studies to support a Rubdium-82 generator or the proximity to a cyclotron that is needed with $\mathrm{N}-13$ ammonia. Finally, the longer half-life of $\mathrm{F}-18$ allows for exercise testing, which is less practical with $\mathrm{N}-13$ ammonia and not practical with Rubdium-82. Flurpiridaz is associated with somewhat higher though still low radiation $(21,22)$.

\section{How to differentiate microvascular disease from 3-vessel disease by PET-MBF?}

The diagnostic and prognostic value of flow and flow reserve measured using dynamic cardiac PET perfusion imaging is now well established $(11,19,20,23-31)$ and presents the advantage for excluding balanced ischemia due to 3-vessel CAD. (19, 20, 23-31,) ASNC guidelines note that quantification of MBF using PET appears to be most useful in the following circumstances (11): "1) patients without a known history of CAD who present with symptoms suspect for myocardial ischemia; 2) patients with known $\mathrm{CAD}$, in whom more specific physiological assessment is desired; 3 ) Identifying [patients with] an increased suspicion for multi-vessel CAD; 4) to assess possible microvascular dysfunction; 5) heart transplant patients when there is a question of transplant vasculopathy".

However, how to distinguish whether impaired flow and flow reserve are due to obstructive CAD versus flow impairments due to endothelial and/or microvascular dysfunction versus varying combinations of both is not easy and, strictly speaking, is not possible when flow measurements are considered in isolation. As such, flow data interpretation must always be considered in context and in conjunction with all available information including history (symptoms, coronary risk factors, known CAD), ECG changes, coronary calcium score, perfusion imaging findings, left ventricular (LV) size and function, right ventricular (RV) uptake and transient ischemic dilation (TID) (20, 23 ).

Is there a cut-off value that predicts obstructive CAD? No; and there are variations in cut-offs for what is defined as normal that vary with different tracers, different analysis software, age, and diffuseness of disease. In general, a global MFR (Myocardial Flow Reserve) $\geq 2.3$ (without regional variability) with virtually all PET perfusion tracers indicates a good prognosis $(11,20)$. Cut-off values for Rubidium-82 and $\mathrm{N}-13$ ammonia have been reported as $\geq 2.0(27,28)$. When flow reserve is above these cut-points, the likelihood of significant obstructive multi-vessel CAD or microvascular disease is lower.

It has been shown that as global MFR decreases, the likelihood of obstructive CAD increases progressively. In one study, the point estimates for the likelihood of 3-vessel obstructive CAD for MFR 
values of $<2.0,<1.5$ and $<1.0$ were $11 \%, 25 \%$ and $48 \%$ respectively. The sensitivities and specificities for MFR cutoff values of $<2.0,<1.5$ and $<1.0$ were 88 and $51 \% ; 80$ and $74 \%$; and 36 and $96 \%$, respectively, for balanced 3-vessel obstructive CAD (27). But these sensitivity and specificity values would indicate that this is not an absolute diagnosis. Accordingly, other information is needed. Any history of known CAD, the presence of coronary calcification and/or regional reductions in flow, point more to indicate obstructive CAD. Their absence points more to microvascular disease. Of note, patients may also have combinations of obstructive CAD in 1,2 or 3 vessels and diffuse atherosclerosis and/or microvascular disease impairing global flow.

To complicate matters further, reduced absolute hyperemic myocardial blood flow and flow reserve with pharmacological vasodilator stress can be due to: multi-vessel obstructive CAD; diffuse atherosclerosis; endothelial dysfunction or microvascular disease; combinations of these; but can also be due to insufficient response to the vasodilator stress (due to caffeine intake or patient specific variances). So-called non-responsiveness should be suspected if global and regional MFR are uniform and at/or about 1.0 with little or no hemodynamic change and no other findings to suggest severe ischemia (i.e., no ECG changes, no change in perfusion, no TID). In such circumstances, the stress portion of the test may need to be repeated either ensuring no caffeine intake, extending the period of caffeine abstinence, or using another pharmacologic stress imaging agent $(20,23)$.

Recent data from small cohorts of patients with CAD have observed that longitudinal flow gradients (measured as the change in flow from the mid to the mid-distal segments of the LV) is correlated to FFR (30) Also, the change in longitudinal flow gradients between stress vs rest (particularly when combined with MFR) provides an accurate means to identify obstructive CAD (31). These data suggest that longitudinal flow gradients may optimize identification of obstructive CAD in the presence of multi-vessel CAD. Others have also examined relative flow reserve (essentially regional stress flow/maximal stress flow) demonstrating that global stress flow identifies well diffuse atherosclerosis while relative flow reserve better identifies significant stenoses. Future studies in patients with microvascular disease are needed to determine if these parameters can truly optimize the distinction of microvascular, endothelial, and diffuse atherosclerotic disease from 3-vessel obstructive CAD or determine their relative importance and contribution to the patient's symptoms or prognosis.

Ultimately integrated structure-function information is required to make the definitive diagnostic distinction between microvascular disease and 3-vessel CAD or define their relative contribution in each patient's coronary vascular disease. Certainly, when global or regional flow reserve is below 1.5 (or 1.7 depending on software and lab), defining anatomy is recommended given the increased risk for these patients $(11,19,20,23,26,28,29)$.

While MBF and MFR have enabled greater ability to detect multi-vessel disease and microvascular disease, there remain challenges in wide spread use of MBF and MFR measurements. Some of these include standardizing measurements between sites and educating ourselves and referring physicians regarding the added clinical value and how to interpret the added flow reserve data with or without multivessel CAD. More prospective multicenter studies are needed to expand our understanding of: the role and importance of microvascular disease and its treatment; the full clinical value of MFR and MBF; and the role of MBF and MFR measurements in selecting and monitoring therapies. 


\section{The scene outside USA: what are the differences and similarities?}

The local practices of imaging in general and nuclear imaging is quite variable outside the United States. $(32,33)$ Just for example there are more than 30 nations involved in the writings of the European Society of Cardiology (ESC) guidelines. In many places in Europe, advanced imaging using state-of-the-art technology including PET is available in academic centers. What is interesting, however, are the subtle differences between ESC and American College of cardiology/American Heart Association (ACC/AHA) guidelines. There is a stronger argument for hybrid imaging in ischemic and non-ischemic cardiac diseases in the ESC guidelines and more emphasis on non-imaging treadmill exercise testing in the ACC/AHA guidelines in ischemic heart diseases (Table 2) $(34,35)$. These differences might disappear in future updates of these guidelines. Parenthetically, almost $1 / 2$ of the submissions to our Journal come from outside the United States and our composition of editors, Board members and publication committee clearly reflect this International collaboration.

\section{Radiation exposure}

The entire field of nuclear cardiology is predicated on the application of a radioactive tracer technique in combination with sophisticated electronic nuclear instruments to image a highly specific signal with superior contrast resolution that allows the differentiation between normal and abnormal myocardial perfusion, metabolism, or innervation. That there is radiation exposure from nuclear imaging has never been disputed, given the fundamental basis of using low doses of radioactive tracers to gain insight into normal and abnormal cardiovascular physiology and disease processes. The concept of applying the ALARA (as low as reasonably achievable) principle is to inject the lowest radiotracer dose possible that allows the acquisition of high quality images which can be interpreted reliably without sacrificing diagnostic accuracy (36-38).

The calculation of radiation risk is imprecise and it represents extrapolated estimated risk from epidemiologic data to the clinical setting (36). As such, there is an ongoing debate regarding the true incremental risk to subjects exposed to low doses currently administered in cardiovascular procedures. There is uncertainty of the dose-response relationship in this lower diagnostic range radiation doses, as well as tissue-specific reparative responses. Given the uncertainty, any ability to reduce radiation by any amount would be a positive advancement and consistent with the principle of ALARA. A study by the IAEA (International Atomic Energy Agency) has documented regional variations world-wide (37). There are many ways to lower the radiation exposure including the use of stress- only imaging when applicable, newer SPECT hard-wares and soft wares, PET imaging, and more importantly appropriate utilization. A recent paper provided a thorough analysis and modeling of the radiation risks (38). It is understood that no discussion on risk is complete without acknowledging the tremendous contributions of nuclear cardiology imaging to patient management which explains why despite all new developments it remains the most popular non-invasive stress test.

\section{Special issues with the new cameras? Is attenuation correction needed? Are there normal databases and are these specific to each camera system?}

The initial reports on the a truly revolutionary change in the design of SPECT detectors to use cardiac-centric solid-state detectors with pinhole $(39,40)$ or parallel-hole collimators have been followed with research reports confirming their value. In addition to using new detectors and/or new collimators, the 
general concept is to image the LV in the center of the field of view to allow more but smaller detectors or magnification of the LV image in the crystal to significantly increase count sensitivity (41). Compared to conventional SPECT, these new systems together with newer reconstruction software that incorporates resolution recovery, noise modeling, with or without attenuation correction yield images with higher contrast and spatial resolution, acquired in shorter time and/or with reduced radiation doses to the patient (42). This advancement in instrumentation and software yields SPECT studies with higher image quality, reduced radiation, and increased efficiency. Similarly, quantification of global and regional LV function has been shown to correlate well (43) with conventional devices even when using lower doses and faster acquisition times with the new cardio-centric devices $(44,45)$. These benefits do come at a higher cost compared to conventional dual head parallel-hole SPECT systems and may be a lower specificity, though this was noted in the early experience (46).

These cardio-centric cameras require centering the LV in the center of a somewhat reduced field of view for optimal results (47). Moreover, for accurate relative perfusion quantification, the higher image contrast resolution of these new cardio-centric systems requires the use of myocardial perfusion normal databases specific to the device or at least from a general class of higher contrast resolution images (42, 48). Just like with standard SPECT cameras, two position imaging (supine/prone or upright/supine) increases the specificity of detecting patients with evidence of myocardial hypo-perfusion, compared to one position imaging (49). When available, attenuation correction has the advantage that it works in concert with the higher energy resolution of the solid-state detectors and the resolution recovery and noise modeling reconstruction to yield more accurate voxel values than two position imaging, and has the potential to provide absolute radiotracer concentrations. Preliminary data suggest feasibility of flow quantification with existing tracers.

\section{Hybrid SPECT/CT and PET/CT}

Hybrid PET/CT or SPECT/CT systems permit simultaneous coronary anatomic and physiologic radionuclide assessment in a single study. Most PET scanners currently used for MPI are hybrid PET/CT systems which can perform CT assessment of coronary anatomy. SPECT/CT systems are also available, but currently make up only a small fraction of the equipment used for SPECT-MPI. The hybrid systems can evaluate the presence and magnitude of CAD using coronary artery calcification (CAC) or coronary CT angiography (CCTA) $(50,51)$

A highly effective and practical application of hybrid PET-SPECT/CT systems is the performance of MPI and CAC scanning in a single setting. CAC provides essentially a "no cost" add on to MPI and is performed with minimal additional radiation to the patient. This combination is complementary to MPI in four important ways.

First, a limitation of stress MPI, whether performed with PET or SPECT, is that regional stress perfusion abnormalities are inducible only in coronary arteries with hemodynamically significant lesions. This approach fails to identify patients with subclinical atherosclerosis in whom aggressive medical and lifestyle modification might prevent subsequent cardiac events. Most patients undergoing MPI have normal examinations (52), and when they are normal, the test results often do not alter patient management. The degree to which this occurs limits the potential of the MPI studies to provide value in our increasingly costconscious era. CAC provides a marker of the extent of calcified coronary atherosclerosis in an individual 
patient, overcoming the limitations of population-based global risk scores. By affording the simultaneous assessment of the presence of coronary atherosclerosis as well as of myocardial ischemia, the finding of an abnormal scan can lead to beneficial changes in therapy and greater patient compliance with therapeutic recommendations (53).

Second, the addition of CAC scanning to MPI improves risk stratification. The presence of CAC and MPI abnormality is synergistic with event rate rising with each increment of abnormal CAC scan categorization (54). While SPECT MPI assessment of ischemia is an excellent test of short-term prognosis, CAC scanning provides better discrimination of long-term risk, due to its assessment of non-obstructive CAD. More than a decade ago, it was recognized that high CAC scores are common in patients with normal SPECT MPI (55). The short-term risk was found to be low in such patients (52); however, subsequent longer-term follow-up demonstrated substantial risk of cardiac events $(54,58)$. The extent of CAC also improves risk assessment of patients with abnormal MPI examinations $(54,58)$.

Third, the combination of CAC with MPI can also lead to increased certainty in scan interpretation and accuracy in detection of hemodynamically significant stenosis. This benefit is particularly useful when faced with borderline MPI abnormalities or when there is discordance between the nuclear MPI results and clinical or ECG responses to stress (55).

Fourth, the extent of CAC abnormality provides a more accurate assessment of the likelihood of obstructive CAD than consideration of age, sex, and risk factors in asymptomatic patients (59). When CAC is absent, the likelihood of CAD is generally low. In this circumstance, borderline findings or discordant MPI results can be weighed toward normal in reporting and in treatment recommendations. Extensive CAC indicates an intermediate to high likelihood of obstructive CAD. When found in a patient with borderline or discordant MPI findings, extensive CAC can be useful in raising the possibility not only of the presence of obstructive CAD but of the potential of high risk CAD (60).

Which patients would benefit from hybrid imaging with combination of CAC with MPI? The above considerations would suggest that it may be all patients without known CAD.

Coronary CTA can also be performed during the MPI examination using recent generation PET/CT systems that have minimum of 64 detector rows and rotation time to produce adequate CTA studies. Sequential testing, MPI followed by CTA in selective patients with documentation of inducible ischemia is also an option. Due to the ability to define the presence, location, and degree of coronary stenosis, CCTA can be more definitive than CAC scanning in guiding management of patients with borderline or discordant MPI results as well as in further assessment of the need for invasive angiography patients with mild degrees of ischemia. The combination of testing essentially eliminates a well-described problem of relative perfusion defect assessment of MPI—the potential failure to identify patients with high risk CAD due to balanced reduction in coronary blood flow (61). To a degree, this problem is averted with PET MPI, as noted above, by addition of absolute quantitation of myocardial perfusion (62); however, also as noted, diffuse reduction of flow reserve, a marker of increased risk, can be seen not only in patients with extensive ischemia from epicardial CAD but also in the presence of microvascular disease. When diffuse flow reduction is observed by PET, further anatomic assessment for this clinically important distinction often becomes necessary. 
Studies have evaluated hybrid PET-MPI/CTA, with both studies performed in the same setting, with respect to prediction of hemodynamically significant stenosis defined by invasive angiography in combination with FFR $(24,63)$. These studies have demonstrated higher accuracy of the combination than with either modality alone. However, because it is difficult to predict in advance whether a patient will need both studies, the practicality and cost-effectiveness of the routine combination of the two studies is questionable. If a single test provides sufficient information to accurately guide patient management, the routine performance of both tests would have resulted in unnecessary costs and radiation. Current opinion is that this routine coupling of MPI with CCTA is not likely to become routine.

Coronary CTA is increasingly being used as the initial test in patients with suspected CAD. However, it is important to point out that anatomic-based CT strategy identifies a significant number of patients with indeterminate coronary CTA lesions (between 30\% and 70\%) requiring additional physiologicbased imaging studies (accruing additional cost and radiation risk). On the other hand, an abnormal initial physiologic-based study (SPECT or PET) would direct the patient to coronary angiography for both diagnostic confirmation of the coronary artery lesion and therapeutic intervention during the same encounter averting unnecessary radiation and contrast from a diagnostic coronary CTA.

Advantages of coronary CTA over a standard of care approach that included stress testing was suggested by the SCOT-Heart trial $(63,64)$. In that trial, a substantial change in preventive therapies in the CCTA arm was noted and likely played a significant role in the trial findings. In the PROMISE trial, which compared coronary CTA to stress testing, there was an increase in statin and aspirin use in coronary CTA arm as well as improved lifestyle behavior. If CAC scanning had been performed along with stress testing, the use of preventive therapies may have been similar. Potentially, routine coupling of CAC scanning with SPECT- or PET-MPI by hybrid scanning, by providing the assessment of coronary atherosclerosis, could allow MPI to be more competitive with coronary CTA. This important consideration as to how hybrid CAC/PET or SPECT MPI would fare regarding "value" when compared to CTA has not been studied and would be of great interest. Several molecular imaging techniques are now in the clinic and others are used as companion for clinical trials and drug development. Many more techniques are in the pipeline.

\section{Training and the imager of the future}

Rapid technological developments in echocardiography, nuclear cardiology, cardiac computed tomography (CCT) and cardiac magnetic resonance (CMR), and the expanded applications of these technologies to the patient with cardiovascular disease, has necessitated a rethinking of the definition and training requirements of the cardiac imaging specialist. Competing considerations influence this prevalent debate, and have resulted in conflicting opinions: the silo approach, of an imager with expertise in a single modality, to the "multi-modality" imager who has expertise in multiple or "all" modalities. The report of a recent think-tank convened by the American College of Cardiology, and the accompanying editorial by the editors of JACC Cardiovascular imaging capture the essence of this debate $(65,66)$.

The required exposure for a trainee within the finite three-year period of a cardiovascular fellowship has expanded considerably, making it difficult to acquire expert level training in multiple imaging modalities without additional training time. Furthermore, the maintenance of a high level of expertise and sustaining credentialing requirements in multiple modalities throughout the course of one's career is difficult. So, can the cardiac imaging subspecialist truly be a multi-modality expert, or should the definition be based on a 
career path dedicated to imaging, with high level of expertise in one or two modalities combined with a broad exposure to other modalities?(66) In this debate, consideration must also be given to the vast array of non-traditional, web-based resources now available to the trainee that make it less time- intensive to acquire a broad exposure to multiple modalities and to maintain competence during clinical practice (67).

Training decisions are heavily influenced by practice setting requirements. The community setting with fewer practitioners favors a multimodality imager and this approach may protect against layered or duplicative testing and overuse of imaging. In an academic setting, individual practitioners working together, each with high-level expertise in one or two modalities may create a multimodality service.

Ultimately, skill sets and thus training, will need to be adapted based on the practice setting (academic or community) and career path (interpretive skills versus a leadership role in an imaging laboratory or research). However, adapting training curricula to individual and market needs is distinct from our quest to optimally define the designation of an advanced cardiac imaging expert. Not everyone who provides interpretive service will qualify for that designation. For the latter, our criteria should be rigorous if we are to maintain our identity as a credible subspecialty. 


\section{References}

1- Iskandrian AE: Myocardial perfusion imaging: Lessons learned and work to be done. 2014; 21: 416

2- Zaret BL. Strauss HW, Martin ND, Wells HP, Flamm MD Jr. -Noninvasive regional myocardial perfusion with radioactive potassium - Study of patients at rest, with exercise and during angina pectoris. N Engl J Med 1973; 288:809-15

3- Strauss HW, Zaret BL, Pieri P, Lahiri A. The Nobel Prize in physics in 1903. J Nuc Cardio, 2017 (in press)

4- Porter, ME, What Is value in health care. N Engl J Med 2010; 363: 2477-2481

5- Gibbons RJ. What is the evidence? A call for scientific rigor. J Nucl Cardiol 2017, 24: 625-648

6- Radford NB, DeFina LF, Barlow CE et al. Progression of CAC score and risk of incident CVD. J Am Coll Cardiol Imag 2016; 9: 1420-9

7- Ford I, Shah AS, Zhang R et al. High-sensitivity cardiac troponin, statin therapy and risk of coronary heart disease. J Am Coll Cardiol 2016;68: 2719-28

8- Germano G, Kavanagh PB, Ruddy TD et al. Same patient processing for multiple cardiac SPECT studies. 2. Improving quantification repeatability. J Nuc Cardiol 2016; 23:1442-1453

9- Lin $X, X u H, Z$ hao $X$, et al. Repeatability of left ventricular dyssynchrony and function parameters in serial gated myocardial perfusion SPECT studies. J Nucl Cardiol 2010; 17:811-6.

10- Zhou Y, Faber TL, Patel Z, et al. An automatic alignment tool to improve repeatability of left ventricular function and dyssynchrony parameters in serial gated myocardial perfusion SPECT studies. Nuclear Medicine Communications 2013, 34:124-129

11- Dilsizian V, Bacharach SL, Beanlands SR, et al. ASNC Imaging Guidelines/SNMMI Procedure Standard for Positron Emission Tomography (PET) Nuclear Cardiology Procedures. J Nucl Cardiol 2016 23:1187-226

12- Gewirtz H, Dilsizian V. Integration of Quantitative PET Absolute Myocardial Blood Flow in the Clinical Management of Coronary Artery Disease. Circulation 2016; 133:2180-96.

13- Tonino PA, De Bruyne B, Pijls NH et al. FAME Study Investigators. Fractional flow reserve versus angiography for guiding percutaneous coronary intervention. N Engl J Med. 2009; 360:213-224.

14- Schindler TH, Dilsizian V. PET-Determined Hyperemic Myocardial Blood Flow: Further Progress to Clinical Application. J Am Coll Cardiol 2014; 64(14):1476-8.

15- Bateman TM, Dilsizian V, Beanlands RS, et al. ASNC/SNMMI Position Statement on the Clinical Indications for Myocardial Perfusion PET. J Nucl Cardiol 2016 Oct; 23:1227-31. 
16- Johnson NP, Kirkeeide RL, Gould KL. Is discordance of coronary flow reserve and fractional flow reserve due to methodology or clinically relevant coronary pathophysiology? JACC Cardiovascular Imag. 012; 5:193-202.

17- Johnson NP, Gould KL. Physiological basis for angina and ST-segment change PET-verified thresholds of quantitative stress myocardial perfusion and coronary flow reserve. JACC Cardiovascular Imag. 2011; 4: 990-998.

18- Lee JM, Kim CH, Koo BK et al Integrated Myocardial Perfusion Imaging Diagnostics Improve Detection of Functionally Significant Coronary Artery Stenosis by $13 \mathrm{~N}$-ammonia Positron Emission Tomography. Circulation: Cardiovascular Imag. 2016;9:e004768

19- Gould KL, Johnson NP, Bateman TM, et al. Anatomic versus physiologic assessment of coronary artery disease: role of coronary flow reserve, fractional flow reserve, and positron emission tomography imaging in revascularization decision-making. J Am Coll Cardiol. 2013; 62:1639-1653

20- Dilsizian V. Transition from SPECT to PET Myocardial Perfusion Imaging: A Desirable Change in Nuclear Cardiology to Approach Perfection. J Nucl Cardiol 2016, 23:337-8.

21- Maddahi J, Bengel F, Czernin J et al. Dosimetry, bio-distribution, and safety of Flurpiridaz F 18 in healthy subjects undergoing rest and exercise or pharmacological stress PET myocardial perfusion imaging. J Nuc cardiol 2017 (in press)

22- Yalamanchili $P$, Wexler E, Hayes M, et al. Mechanism of uptake and retention of $18 \mathrm{~F} \mathrm{BMS747158-}$ 02 in cardiomyocytes: A novel PET myocardial imaging agent. J Nucl Cardiol. 2007; 14:782-8.

23- Juneau D, Erthal F, Ohira $\mathrm{H}$, et al. Clinical PET Myocardial Perfusion Imaging and Flow Quantification. Cardiol Clin. 2016; 34:69-85.

24- Kajander S, Joutsiniemi E, Saraste M, et al. Cardiac positron emission tomography/computed tomography imaging accurately detects anatomically and functionally significant coronary artery disease. Circulation; 122:603-613.

25- Hajjiri MM, Leavitt MB, Zheng H, Spooner AE, Fischman AJ, Gewirtz H. Comparison of positron emission tomography measurement of adenosine-stimulated absolute myocardial blood flow versus relative myocardial tracer content for physiological assessment of coronary artery stenosis severity and location. J Am Coll Cardiol Imag 2009;2;751-758

26- Herzog BA, Husmann L, Valenta I, et al. Long-term prognostic value of $13 \mathrm{~N}$-ammonia myocardial perfusion positron emission tomography added value of coronary flow reserve. J Am Coll Cardiol 2009; 54:150-156.

27- Ziadi MC, Dekemp RA, Williams KA, et al. Impaired myocardial flow reserve on rubidium-82 positron emission tomography imaging predicts adverse outcomes in patients assessed for myocardial ischemia. J. Am. Coll. Cardiol. 2011; 58:740-748.

28- Murthy VL, Naya M, Foster CR, et al. Improved cardiac risk assessment with noninvasive measures of coronary flow reserve. Circulation 2011; 124: 2215-2224.

29- Ziadi MC, Dekemp RA, Williams K, et al. Does quantification of myocardial flow reserve using rubidium-82 positron emission tomography facilitate detection of multivessel coronary artery disease? J. Nucl. Cardiol. 2012; 19: 670-680.

30- Valenta I, Antoniou A, Marashdeh W, et al. PET-measured longitudinal flow gradient correlates with invasive fractional flow reserve in CAD patients. Eur Heart J Cardiovascular Imag. 2016 Jun 19. pii: jew116. [Epub ahead of print] 
31- Valenta I, Quercioli A, Schindler TH. Diagnostic value of PET-measured longitudinal flow gradient for the identification of coronary artery disease. JACC Cardiovascular Imag. 2014; 7: 387-96.

32- Mercuri M, Pascual TNB, Mahmarian JJ, et al for the INCAPS Investigators Group. Findings from the IAEA (International Atomic Energy Agency) Nuclear Cardiology Protocols Study. JAMA Intern Med. 2016; 176: 266-269.

33- Einstein AJ, Pascual TNB, Mercuri M et al for the INCAPS Investigators Group. Current worldwide nuclear cardiology practices and radiation exposure: results from the 65 country IAEA Nuclear Cardiology Protocols Cross-Sectional Study (INCAPS). Eur Heart J 2015:36: 1689-1696.

34- Petretta M, Cuocolo A. Comparison of ESC and ACC/AHA guidelines for myocardial revascularization. Are the differences relevant? The European perspective. J Nuc Cardiol 2017;24: 1057-1061

35- Stirrup J, Velasco A, Hage FG. Guidelines in review: Comparison of ESC and ACC/AHA guidelines for myocardial revascularization J Nuc Cardiol 2017;24:1046=1053

36- Laskey WK, Feinendegen LE, Neumann RD, Dilsizian V. Low-Level lonizing Radiation from Noninvasive Cardiac Imaging: Can we Extrapolate Estimated Risks from Epidemiologic Data to the Clinical Setting? J Am Coll Cardiol Imag 2010; 3: 517-24.

37- Shi L, Dorbala S, Paez D et al. Gender Differences in Radiation Dose From Nuclear Cardiology Studies Across the World. J Am Coll Cardiol Imag 2016; 9: 376-384

38- Zanzonico P, Dauer L, Strauss HW. Radiobiology in cardiovascular imaging. J Am Coll Cardiol Imag 2016; 9:1446-61

39- Esteves, FP; Raggi, P; Folks, RD et al. Novel solid-state-detector dedicated cardiac camera for fast myocardial perfusion imaging: multicenter comparison with standard dual detector cameras. J Nucl Cardiol 2009; 16: 927-934

40- Duvall, WL; Croft, LB; Godiwala, T et al. Reduced isotope dose with rapid SPECT MPI imaging: Initial experience with a CZT SPECT camera. J Nucl Cardiol 2010, 17: 1009-1014

41- Garcia EV. Physical attributes, limitations, and future potential for PET and SPECT 2012, J Nucl Cardiol:19 Supplement: 1: S19-S29

42- Fabio EP, Galt JR, Folks RD, et al. Diagnostic performance of low-dose rest/stress Tc-99m tetrofosmin myocardial perfusion SPECT using the 530c CZT camera: Quantitative vs visual analysis. J Nucl Cardiol 2014: 21: 158-165.

43- Bailliez A, Blaire T, Mouquet $F$ et al. Segmental and global left ventricular function assessment using gated SPECT with a semiconductor Cadmium Zinc Telluride (CZT) camera: Phantom study and clinical validation vs cardiac magnetic resonance. J Nucl Cardiol 2014, 21: 712-722

44- Duvall WL, Sweeny JM, Croft LB, et al. Reduced stress dose with rapid acquisition CZT SPECT MPI in a non-obese clinical population: Comparison to coronary angiography. 2012, 19: 19-27

45- Oddstig J, Hedeer, F Jogi J et al. Reduced administered activity, reduced acquisition time, and preserved image quality for the new CZT camera. J Nucl Cardiol 2013: 20 38-44

46- Nudi $F$, Iskandrian AE, Schillaci $O$, et al. Diagnostic accuracy of myocardial perfusion imaging with CZT technology. Systemic review and meta-analysis of comparison with invasive coronary angiography. JACC: Cardiovascular Imag, 2017; 10: 787-94

47- Hindorf C; Oddstig, J; Hedeer, F, et al. Importance of correct patient positioning in myocardial perfusion SPECT when using a CZT camera. J Nucl Cardiol 2014, 21: 695-702. 
48- Miao, L., Kansal, V, Wells, R. et al. Adopting new gamma cameras and reconstruction algorithms: Do we need to re-establish normal reference values? J Nucl Cardiol 2016, 23: 807-817

49- Duvall, W L, Slomka, PJ.; Gerlach, JR.et al. High-efficiency SPECT MPI: Comparison of automated quantification, visual interpretation, and coronary angiography J Nucl Cardiol 2013, 20: 763-773

50- Harrington R, Walsh R, Fuster V. Hurst's the Heart, 14th Edition: Two Volume Set: McGraw-Hill Education; 2017.

51- SNMMI/ASNC/SCCT guideline for cardiac SPECT/CT and PET/CT 1.0., J Nucl Med. 2013; 54:1485-507. PMID: 23781013

52- Rozanski A, Gransar H, Hayes SW, et al. Temporal trends in the frequency of inducible myocardial ischemia during cardiac stress testing: 1991 to 2009. J Am Coll Cardiol 2013; 61:105465.

53- Berman DS, Rozanski A. Value-based imaging: Combining coronary artery calcium with myocardial perfusion imaging. J Nucl Cardiol 2016; 23:939-41.

54- Engbers EM, Timmer JR, Ottervanger JP et al. Prognostic Value of Coronary Artery Calcium Scoring in Addition to Single-Photon Emission Computed Tomographic Myocardial Perfusion Imaging in Symptomatic Patients. Circ Cardiovascular Imag 2016; 9.

55- Berman DS, Wong ND, Gransar H, et al. Relationship between stress-induced myocardial ischemia and atherosclerosis measured by coronary calcium tomography. J Am Coll Cardiol 2004; 44:923-30.

56- Engbers EM, Timmer JR, Ottervanger JP, et al. Sequential SPECT/CT imaging for detection of coronary artery disease in a large cohort: evaluation of the need for additional imaging and radiation exposure. J Nucl Cardiol 2017; 24:212-23.

57- Chang SM, Nabi F, Xu J, et al. The coronary artery calcium score and stress myocardial perfusion imaging provide independent and complementary prediction of cardiac risk. J Am Coll Cardiol 2009; 54:1872-82.

58- Schenker MP, Dorbala S, Hong EC, et al. Interrelation of coronary calcification, myocardial ischemia, and outcomes in patients with intermediate likelihood of coronary artery disease: a combined positron emission tomography/computed tomography study. Circulation 2008; 117:1693700 .

59- Budoff MJ, Diamond GA, Raggi P, et al. Continuous probabilistic prediction of angiographically significant coronary artery disease using electron beam tomography. Circulation 2002; 105:1791-6.

60- Akincioglu C, Belhocine T, Gambhir S, et al. Complementary roles of low-dose SPECT-CT and high-resolution volume CT for detection of coronary artery disease. Clinical nuclear medicine 2008; 33:285-7.

61- Berman DS, Kang X, Slomka PJ, et al. Underestimation of extent of ischemia by gated SPECT myocardial perfusion imaging in patients with left main coronary artery disease. J Nucl Cardiol 2007; 14:521-8.

62- Di Carli MF, Dorbala S, Curillova Z, et al. Relationship between CT coronary angiography and stress perfusion imaging in patients with suspected ischemic heart disease assessed by integrated PET-CT imaging. J Nuc Cardiol 2007; 14:799-809.

63- Danad I, Raijmakers PG, Appelman YE, et al. Hybrid imaging using quantitative H215O PET and CT-based coronary angiography for the detection of coronary artery disease. J Nucl Med 2013; 54:55-63. 
64- CT coronary angiography in patients with suspected angina due to coronary heart disease (SCOTHEART): an open-label, parallel-group, Multi-centre trial. Lancet 2015; 385:2383-91.

65- Williams MC, Hunter A, Shah AS, et al. Use of coronary computed tomographic angiography to guide management of patients With coronary disease. J Am Coll Cardiol 2016; 67:1759-68.

66- Douglas PS, Cerqueira MD, Berman DS, et al. The Future of Cardiac Imaging. Report of a Think Tank Convened by the American College of Cardiology. JACC: Cardiovascular Imag 2016; 9:121123.

67- Marwick TH, Chandrashekhar Y, Narula J. Training in Multimodality CV Imaging. Is There an Inclusive Model? JACC: Cardiovascular Imag 2016; 9:1235-7 
Table 1: Myocardial perfusion imaging: Lessons learned and work to be done (from ref 1)

1- $\quad$ Physiology of the coronary circulation as a building block to perfusion imaging

2- $\quad$ Are the data on sensitivity and specificity valid knowing that severity of stenosis on coronary angiography does not predict flow or fractional flow reserve (FFR)? What is the current role of FFR?

3- $\quad$ Can we have new recommendations on doses and sequences of imaging and stress imaging only?

4- Why is attenuation correction not widely used?

a. Have the newer software programs eliminated the need for attenuation correction?

b. Does early imaging with the newer cameras decrease sub-diaphragmatic activity and the need for attenuation correction?

c. What is the current processing algorithm of choice?

5- Is hybrid stress testing underused or overused? Are there special populations that have yet to be addressed?

6- $\quad$ How do we integrate nuclear and nonnuclear data into personalized medicine in guiding patient management?

7- Should the newer cameras replace the conventional ones or are there still unanswered questions?

8- $\quad$ What is the interaction between scar and ischemia with left ventricular (LV) ejection fraction (EF) and volumes?

9- $\quad$ How does one compare summed rest score (SRS), summed stress score (SSS), and summed difference score (SDS) by visual or automated method and polar maps-derived indices from one commercial software to another?

10- $\quad$ Are predictors of sudden death comparable to those predicting death of any cause or cardiac death?

11- Is viability testing clinically useful and how should this data be used in clinical decision-making?

12- What about non-perfusion variables?

13- Do statistical analyses correct or create problems? Do advanced statistical methods open novel domains in the study of nuclear cardiology?

14- How far is cardiovascular molecular imaging from mainstream clinical use?

15- What is a model nuclear cardiology laboratory in the next decade? Will it include SPECT or PET or both and in what degree?

16- Does artificial intelligence have a role in the modern nuclear cardiology laboratory? 
Table 2: Comparison between ESC and ACC/AHA guidelines in CAD

\begin{tabular}{|c|c|c|c|}
\hline & & ESC & ACC/AHA \\
\hline \multirow[t]{8}{*}{ Detection of CAD } & $<15 \%$ probability & IIIA-stress MPI and echo & $\begin{array}{l}\text { IIIC-stress MPI, echo, M } \\
\text { rest ECG is interpretable }\end{array}$ \\
\hline & & $\begin{array}{l}\text { IIIC-Stress MRI, PET, } \\
\text { Hybrid, CTA }\end{array}$ & \\
\hline & $15-85 \%$ probability & A-stress MPI, MRI, PET & IA-TET \\
\hline & & IIA- CTA & $\begin{array}{l}\text { IB-exercise MPI or echo } \\
\text { ECG is interpretable }\end{array}$ \\
\hline & & IIB-hybrid imaging & $\begin{array}{l}\text { IB-Pharm MPI or echo if } \\
\text { patient cannot exercise }\end{array}$ \\
\hline & & & $\| A-C T A(L O E B)$ \\
\hline & & & IIB-CAC (LOEC) \\
\hline & $>85 \%$ probability & IA-invasive angiography & IA-invasive angiography \\
\hline \multirow[t]{2}{*}{ CR } & DM & $\begin{array}{l}\text { IIA-PCI for syntax score } \\
<22 \text { (LOE B)o }\end{array}$ & IA-CABG \\
\hline & No DM & $\begin{array}{l}\text { New generation stents as } \\
\text { default in all patients }\end{array}$ & $\begin{array}{l}\text { Bare metal stents for hig } \\
\text { bleeding risk, inability to } \\
\text { comply with DAPT }>12 \\
\text { months, anticipated non. } \\
\text { cardiac surgery }<12 \text { mor }\end{array}$ \\
\hline \multirow[t]{2}{*}{-After CR } & DM & $\begin{array}{l}\text { Imaging is preferred over } \\
\text { TET for new or worsening } \\
\text { symptoms }\end{array}$ & $\begin{array}{l}\text { TET or imaging for new } \\
\text { worsening symptoms }\end{array}$ \\
\hline & & $\begin{array}{l}\text { For asymptomatic, IIC }>2 \\
\text { years for } P C l \text { and }>5 \text { years } \\
\text { for } C A B G\end{array}$ & $\begin{array}{l}\text { For asymptomatic, IIA-pI } \\
\text { silent ischemia, high risk } \\
\text { patients or incomplete C } \\
\text { (LOE C) }\end{array}$ \\
\hline
\end{tabular}

Abbreviation

PET: Positron emission tomography, CTA: computed tomography angiography, MRI: Magnetic resonance imaging, TET: treadmill exercise testing, CAC: coronary artery calcium, CAD: Coronary artery disease, ESC: European congress of cardiology, ACC: American college of cardiology, AHA: American heart association MPI: Myocardial perfusion imaging, DM: diabetes mellites, PG: Coronary artery bypass grafting, DAPT: dual antiplatelet therapy, LOE: level of evidence, CR: coronary revascularization 


\section{Legends -}

Figure 1: Single photon emission tomographic (SPECT) myocardial perfusion images (MPI) (post stress and rest) showing a large reversible abnormality in the left panel and a large fixed abnormality in the right panel

Figure 2: Polar maps from stress/rest SPECT MPI obtained from the one patient. Top panel: before medical treatment; middle panel: after treatment and lower panel: after discontinuation of treatment. The patient exercised to the same heart rate and achieved similar METS at all 3 examinations. Though the perfusion abnormality decreased after treatment, there was no change in exercise time but the patient felt subjectively improved. He felt worse after discontinuation of treatment with the larger perfusion defect. 
Fig 1

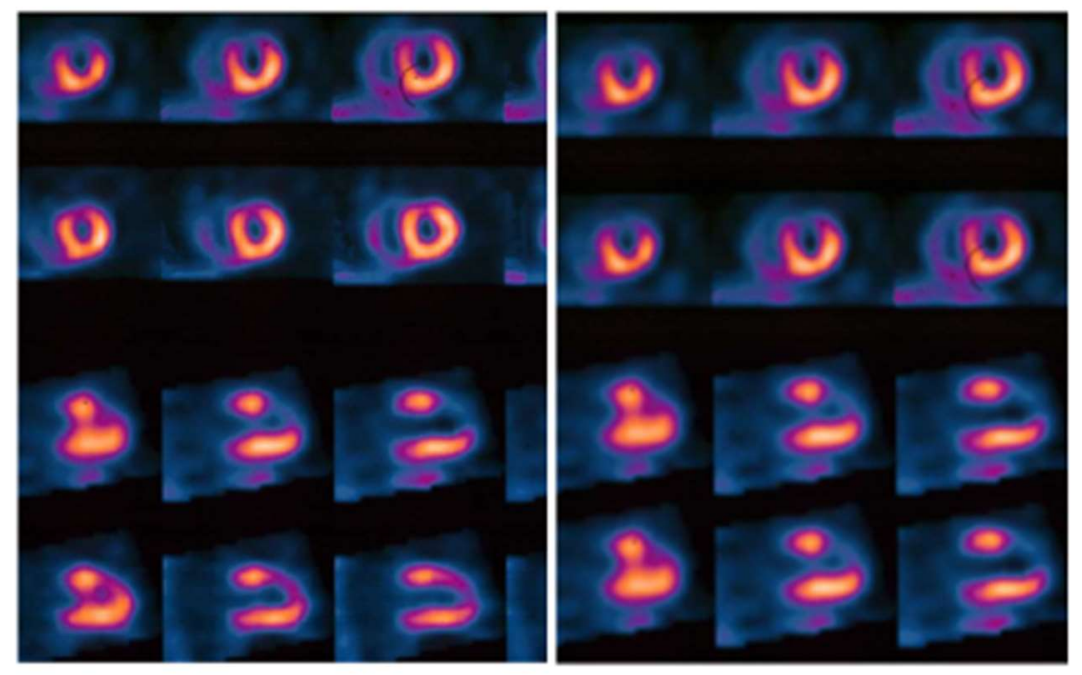

Fig 2

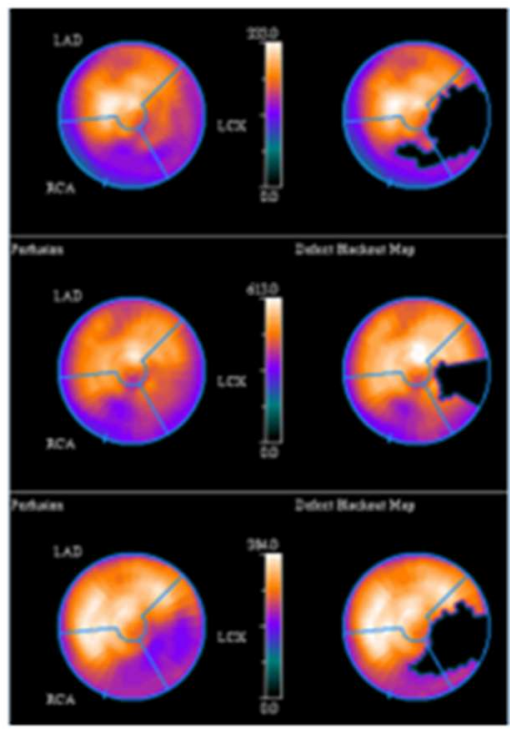


Fig 1

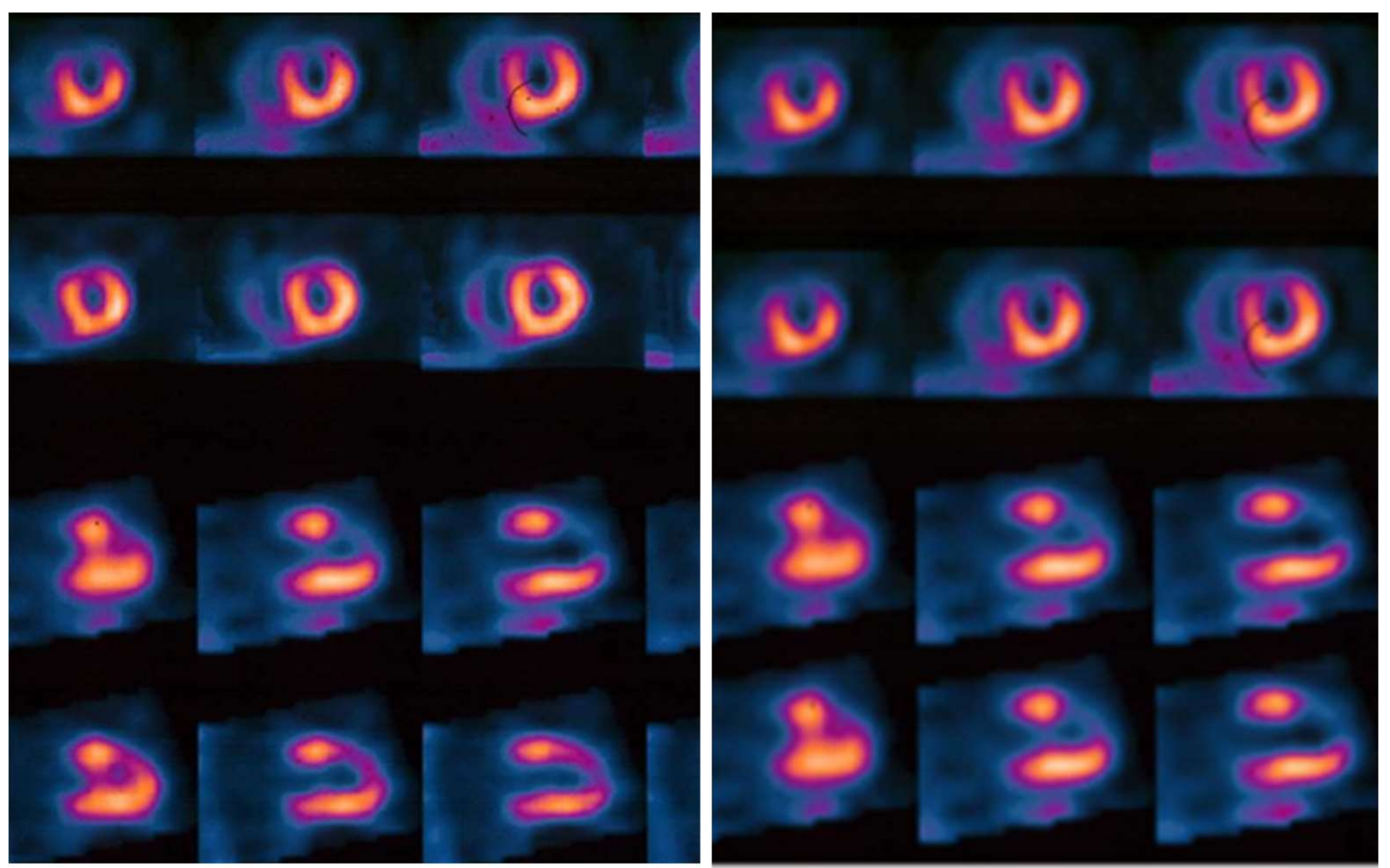


Fig 2

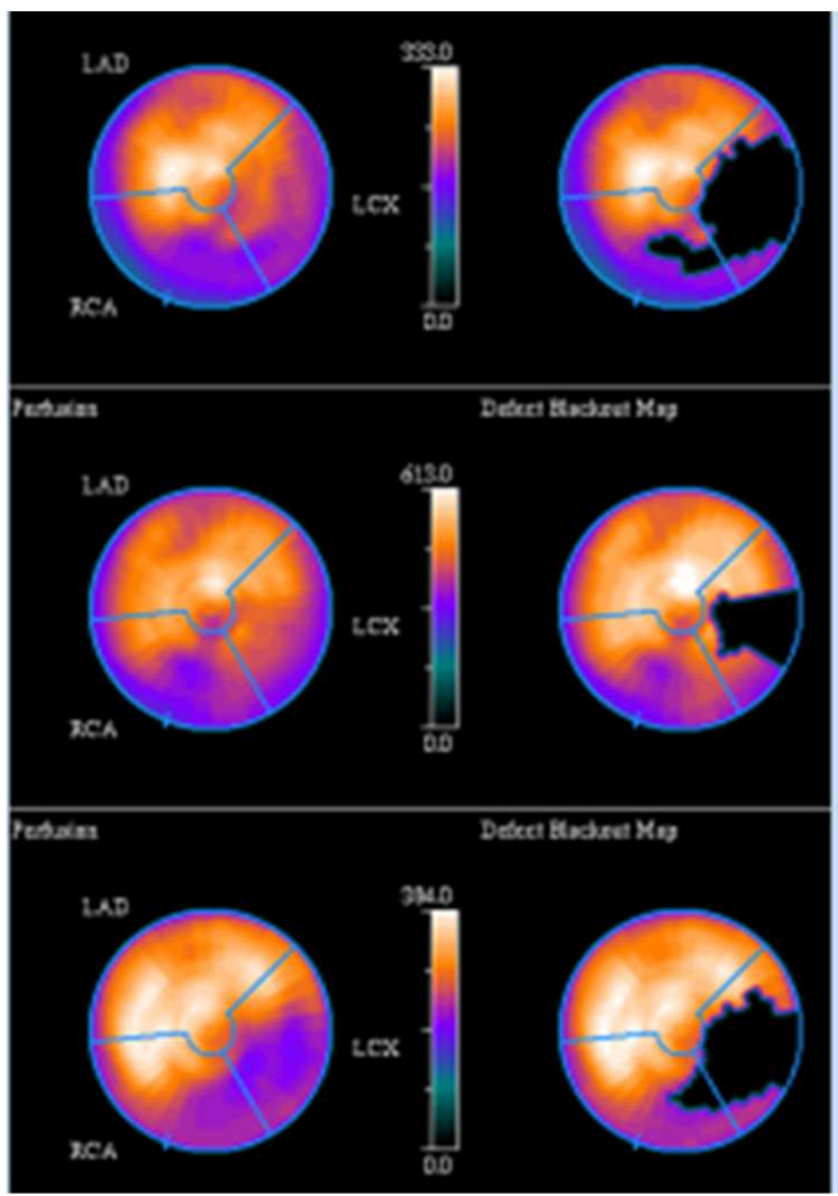

